Jap. J. Physiol., 21, 419-430, 1971

\title{
TONIC VIBRATION REFLEX IN HUMAN AND MONKEY SUBJECTS
}

\author{
Saburo Homma, Kenro Kanda and Shiroh Watanabe \\ Department of Physiology, School of Medicine, Chiba University, Chiba, Japan
}

\begin{abstract}
Summary 1. Both in human subjects and monkeys, the tonic vibration reflex, TVR, was observed during vibratory stimulation of the muscle. In the latter, TVR reaches its maximum soon after vibratory application.

2. In the monkey, unitary EMG was recorded during vibratory stimulation and nonsequential interspike interval histograms were obtained. Intervals of unitary EMG were shown to occur on the principle of integer multiplication of the vibratory cyclic time.

3. A gradual increase or decrease of TVR during vibratory stimulation in the human subject or the monkey is effected by the EMG discharges of shorter or longer firing intervals which occur on this principle.

4. Both in human subjects and monkeys, the TVR becomes larger with the higher range of vibratory frequency. Beyond a certain vibratory frequency the TVR shows a gradual decrease. The relationship between the TVR and the vibratory frequency was called the TVR $-f$ relation. There is an optimal frequency of vibratory stimulation for producing the largest TVR tension.

5. The size of the reflex tension from TVR $-f$ relation depends on the discharge frequency of the unitary EMG, which occurs according to the principle of the integer multiplication of original vibratory cyclic time.
\end{abstract}

It has been demonstrated in cat muscle that primary spindle endings are much more sensitive to muscle vibration than secondary endings, their maximal response frequency being around $400 \mathrm{~Hz}$ (Granit and HeNATSCH, 1956; Bessou and LAPort, 1962; BiAnConi and VAN Der Meulen, 1963; Brown et al., 1967). It has also been reported by several authors that group Ia afferent discharges elicited by sinusoidal stretch activate the spinal motoneuron monosynaptically (MATthews, 1966; Homma et al., 1967).

Vibration of the muscle tendon in awake human subject causes a gradual

Received for publication March 17, 1971

本間三郎, 神田健郎, 渡部土郎 
increase of muscle active tension, which has been designated as tonic vibration reflex, TVR (HAGBARTH and EKLUND, 1966a, b). In our recent studies on the time course of such activity, tension production in human TVR reaches its maximum just before cessation of vibration, while in the anesthetized monkey and cat experiments, TVR readily reaches its maximum soon after vibratory application and shows gradual waning. A gradual increase of TVR during vibratory stimulation is also known to occur to an even greater extent in the unanesthetized and decerebrated cats.

The previous paper reported in detail the mode of monosynaptic coding by the observation of both unitary EMG and motoneuronal spikes evoked during sinusoidal stretch of cat muscles (Homma et al., 1971).

Motoneuron spikes are known to be coded by group Ia afferents during sinusoidal stretch. Minimum displacement of sinusoidal stretch, $\boldsymbol{\Delta} L$, which was necessary to elicit a certain frequency of motoneuronal spikes, was obtained at various sinusoidal frequencies, $f$. Such relationships between $\Delta L$ and $f$ were obtained previously (Homma et al., 1970).

The present paper deals with studies on the mode of coding of the efferent side from afferent impulse during vibratory stimulation both in man and the monkey and on comparisons of TVR- $f$ diagrams between human and primate subjects, the former in awake and the latter in anesthetized states.

\section{METHOD}

Experiments were done on 8 awake normal human subjects and 4 monkeys weighing $4.0-6.5 \mathrm{~kg}$ under light Ketalar* $(10-15 \mathrm{mg} / \mathrm{kg})$ and very light chloraloseurethane anesthesia. A Hagbarth type vibrator was used in all the experiments. The vibrator is a precise miniature D.C. motor which drives changeable eccentric weights weighing between 5.0-25 grams. Rotation of eccentric weights initiates vibration. The frequency precisely corresponds to one sixtieth of the motor's rpm. The whole vibration system was tightly covered with a plastic film to prevent accidental shock currents. The vibrator was attached to the patellar or Achilles tendon of the human and monkey subjects which were in the relaxed sitting position. The frequency of vibration could be changed by using a current source of adjustable D.C. voltage. The frequency of vibration was monitored by a built-in magnetic pick-up device. This signal was fed into a digital frequency counter. Adjustment of vibratory intensity was based on the size of the experimental subject. This was done by using 22 different sizes of eccentric weight; lighter weights were used in the monkey experiments.

In both the human and monkey experiments the knee joint was in the flexed position of $90^{\circ}$ degree and was fixed on both sides by soft pads attached to adjustable bars. Preparatory knee jerk tests were made using manual tendon taps to

\footnotetext{
* Sankyo Pharmaceutical Co., Tokyo
} 
find the most appropriate angle of the knee joint and site of tendon for the vibratory application before the Hagbarth type vibrator was fixed to the tendon. The TVR of the quadriceps femoris muscle was recorded throughout all experiments. The experimental foot was fixed to a plastic plate which was suspended by a steel plate attached to a strain gauge. In the monkey experiments the depth of anesthesia was monitored by intermittent recording of the electroencephalogram because initiation of TVR was dependent on the depth of anesthesia, as is described in a later section of this report.

\section{RESULTS}

\section{Tonic vibration reflex (TVR) in the human and monkey}

The patellar tendon was vibrated in man and monkey and tension production was monitored during extension of the extremity almost isometrically, as mentioned above. In the human subject, three different eccentric weights were used to obtain different intensities of vibration. The heavier eccentric weights caused larger TVR tension. As seen in Fig. 1, the time courses of human and monkey subjects are evidently different: tension production reaches its maximum just before cessation of vibration in human experiments, while in monkey experiments the TVR readily reaches its maximum soon after vibratory application and shows gradual waning. Common characteristics exist only in that both are tonic in their general appearance and that heavier weight initiates more pronounced reflex activity. The monkey in Fig. 1B was lightly anesthetized by chloralose-urethane because stable registration of reflex activity was not possible when the monkey was fully awake. A pronounced TVR could be best observed when the monkey

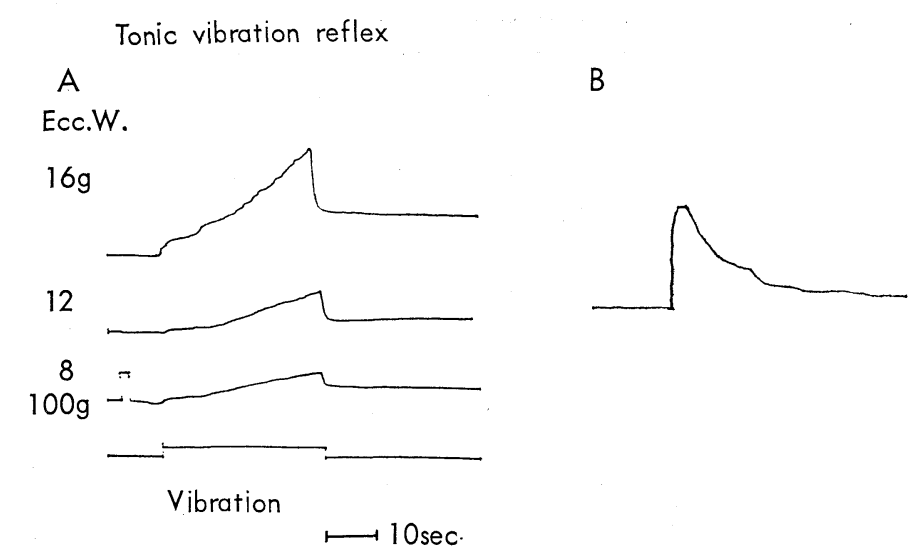

Fig. 1. TVR in human subject (A) and monkey (B). A: Three different eccentric weights were used. The largest eccentric weight, $16 \mathrm{~g}$, caused the largest TVR tension initiated in this case. 
was awakening from anesthesia. After this critical stage, the TVR was often interrupted by the artifact of voluntary movement and stable recording of TVR was impossible. Therefore it was necessary to monitor the appropriate stage of anesthesia by EEG recording. In most of the present experiments TVR was most readily observed with beta electroencephalographs.

The difference between TVR time courses of human experiments and monkey experiments is probably attributable to the difference in the subjects' states of conciousness, the former being fully awake, the latter lightly anesthetized. Since anesthesia or abolition of supraspinal innervation has an overwhelming influence on the stretch reflex, such influence has been suggested to be effected through supraspinal adjustment in the gamma acceleration. Experiments on the decerebrate animal have justified such conclusions (GRANIT et al., 1956).

\section{TVR and EMG spike discharge}

During vibration, muscle spike discharges of the quadriceps femoris in monkeys were recorded with a coaxial needle electrode inserted into the muscle.

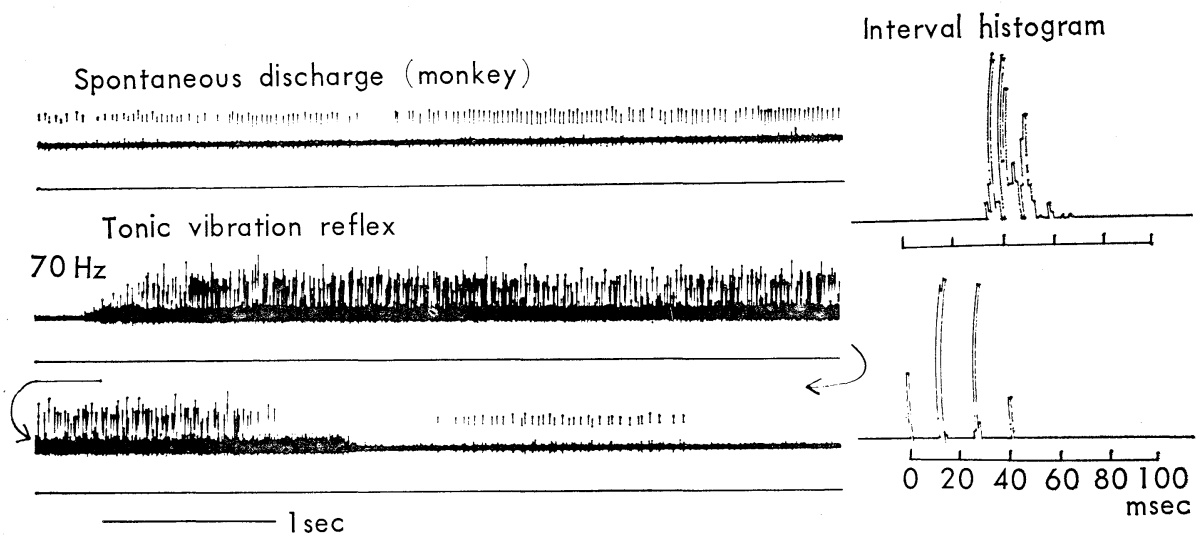

Fig. 2. Spontaneous discharge and spike discharge during vibratory stimulation in the quadriceps femoris muscle. Each nonsequential interspike interval histogram is indicated on the right. The lower trace in each record is the simultaneous monitoring of vibration. The lower two sets are a continuous record during and after $70 \mathrm{~Hz}$ vibration.

Figure 2 shows a continuous record of muscle spike discharge during $70 \mathrm{~Hz}$ vibration. From the onset of vibration the spike discharge decreased rather gradually. Following a vibration of 6 sec duration, spontaneous discharge was seen for about $1.5 \mathrm{sec}$ (Fig. 2). The uppermost trace in Fig. 2 shows the spontaneous discharge recorded with the same needle electrode. Pre- and postvibratory spontaneous discharges are similar in their discharge frequencies. To the right of the figure, nonsequential interspike interval histograms of spontaneous and 
TVR discharges are shown. Interspike intervals in the former (upper histogram) are in the range between 30 and $50 \mathrm{msec}$, but EMG spikes during vibration are quite different (lower histogram). If the data with a very short interval is excluded, the muscle fired with the three kinds of interval ranges between 12-14, 26-28 and 41 msec. Differences between each peak are around $14 \mathrm{msec}$, which approximately corresponds to the cyclic time of $70 \mathrm{~Hz}$. It seems that any muscle spike interval elicited by vibration is invariably equal to the integer multiplication of the original vibration frequency.

Spontaneous discharges are known to fire with longer intervals than the discharges elicited by vibration. Such spontaneous activity with relatively long intervals is understood as motoneurons irrelevance to vibratory afferent input and may suggest that firings of longer intervals include information which is not directly coded by the participating vibratory afferents and that they may fire at their own intrinsic firing frequency.

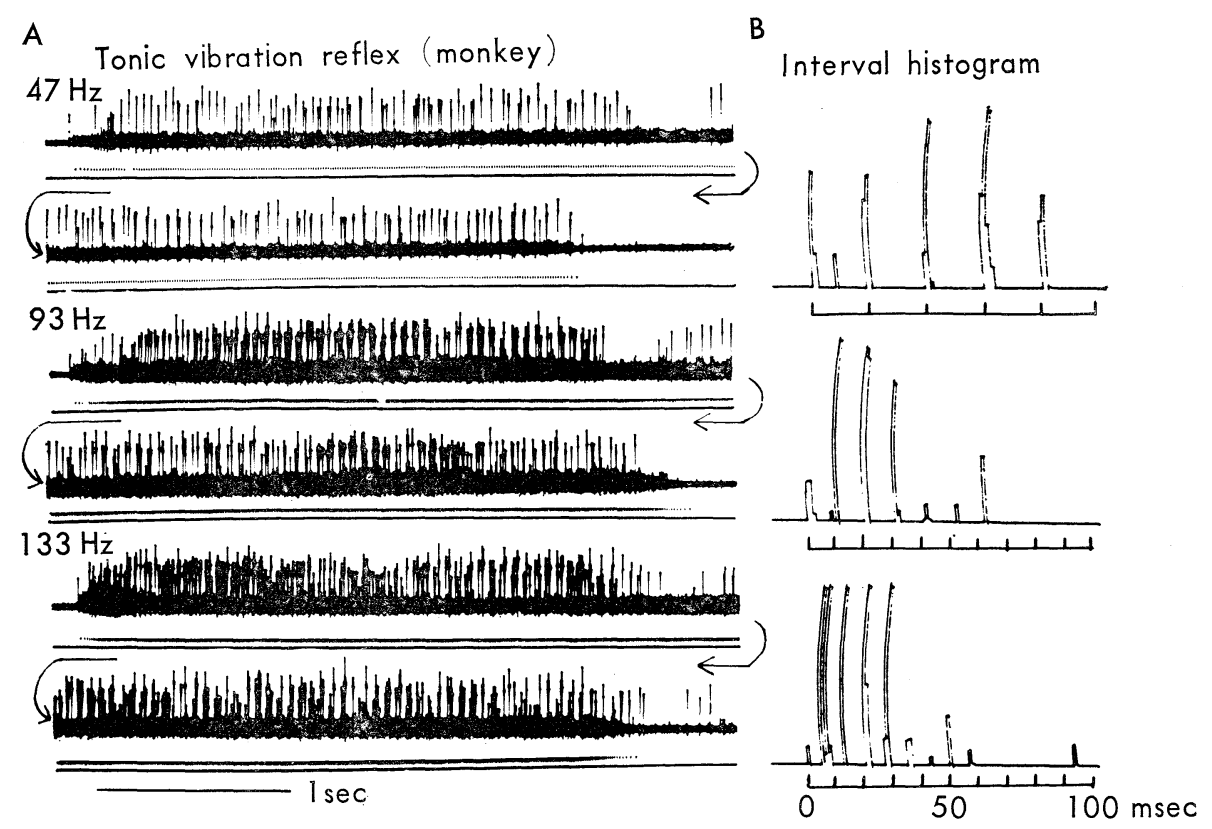

Fig. 3. A: Continuous record of TVR in monkey quadriceps femoris muscle during vibratory stimulation of three different frequencies. B: Each interval histogram is shown. Differences between each interval were $20 \mathrm{msec}$ at $47 \mathrm{~Hz}, 10 \mathrm{msec}$ at $93 \mathrm{~Hz}$ and $7 \mathrm{msec}$ at $133 \mathrm{~Hz}$. The interval difference was equal to the cyclic time of vibration used.

The EMGs shown in Fig. 3 are continuous records of the TVR observed in the quadriceps femoris muscle. The vibratory frequency was set at 47, 93 and $133 \mathrm{~Hz}$, as shown in the uppermost to the bottom trace, respectively. The duration of each vibratory stimulus was approximately $7 \mathrm{sec}$. In this particular 
experiment the EMG always diminished $3 \mathrm{sec}$ after vibration and shows a pause of $0.5 \mathrm{sec}$ for unknown reasons. Nonsequential interspike interval histograms were obtained for respective EMG recordings, which are shown to the right of the original records. An interesting fact revealed by the histograms is that the EMG fired most frequently at a certain interval with statistical certainty. This is very analogous to the findings shown in Fig. 2. The frequencies of the EMG themselves refer to excitation of the muscle and accordingly, the frequency of spinal motoneuron activity which innervates the muscle fiber under observation. During a vibration of $47 \mathrm{~Hz}$, intervals were 20,40, 60 and $80 \mathrm{msec}$ except for very short intervals. Differences, at $97 \mathrm{~Hz}$ were about $10 \mathrm{msec}$ and at $133 \mathrm{~Hz}$ about $7 \mathrm{msec}$. It is clear that each is equal to the cyclic time of the applied vibration. After such statistical analysis it is safe to say that frequencies of EMG discharges during vibratory stimulation closely correspond to the integer multiplication of the cyclic time in msec of the applied vibration.

In cats, the reflex discharge was recorded from a functionally single fiber of $\mathrm{L}_{7} \sim \mathrm{S}_{1}$ ventral root during muscle vibration. The histogram analysis of this ventral root was done by the parameter of the interspike intervals. It was already known that the intervals were scattered around integer multiplication of vibration frequency. For example, at $100 \mathrm{~Hz}$ the vibration frequency of the interspike intervals were $60,70,80$ or $90 \mathrm{msec}$ and each difference was $10 \mathrm{msec}$ (Homma et al., 1971).

During muscle vibration, the excitatory postsynaptic potentials, EPSPs, are initiated at the motoneuronal membrane precisely synchronous to each sinusoidal stretch, especially in the very high frequency range of muscle vibration. Some EPSPs reach the critical threshold level for the firing of a motoneuron. Therefore it is reasonable that the interspike intervals during vibration are phased by the integer times of vibration frequency (Homma et al., 1970a).

It is then clear that TVR muscle tension is produced by the muscle spike discharge occurring by such interspike intervals. When the muscle tension increases gradually, as in human subjects, it can be assumed that the intervals decrease or are shortened step by step by the integer multiplication of vibration frequency. On the other hand, TVR of the anesthetized monkey shows a gradual decrease after a rather abrupt initiation during vibration. During this decreasing phase, a decrease of muscle spike discharge may be effected through an increase of interspike intervals at integer multiplication of vibration frequency.

\section{TVR and vibratory frequency}

Human subjects were used to study the relationship between the size of the TVR (in grams) and vibratory frequency. The results of this experiment are shown in Fig. 4.

The size of the TVR was measured at the maximum phase of the reflex activity during vibratory stimulation which was continued for $30 \mathrm{sec}$. As seen in 


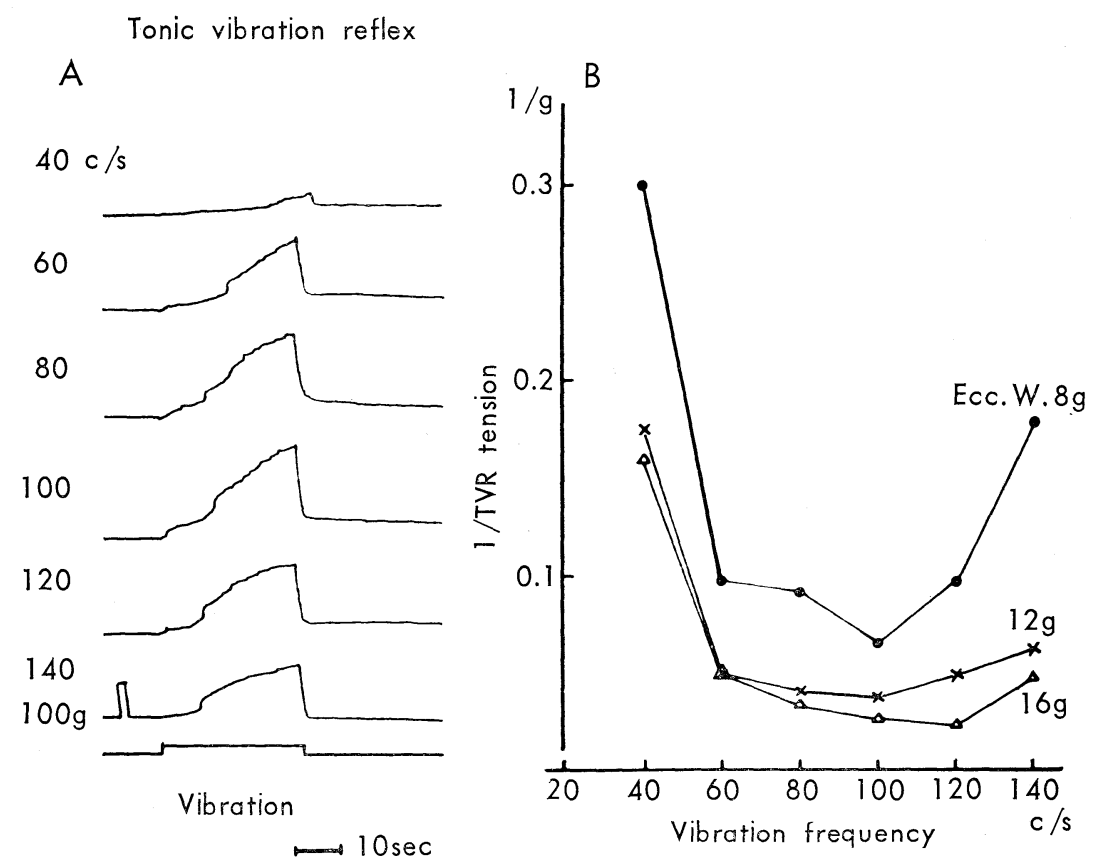

Fig. 4. TVR and vibratory frequency in human subjects.

A: TVR was relatively smaller in both lower and higher ranges of vibratory frequency. B: Relations between reciprocals of maximum tension and vibratory frequency. Results from three different eccentric weights are plotted.

Fig. 4A, the size of the TVR is relatively smaller in both the lower and higher vibratory frequency ranges, showing the largest around $100 \mathrm{~Hz}$.

Although the eccentric weight was not changed during any one series of experiments, acceleration changed arbitrarily with varied vibratory frequencies. The diagram in Fig. 4 is the result of the overall effect of several or more masked vibratory parameters, vibratory frequency, excursion, direction, accerelation or others. To understand the reflex effectiveness of the vibration, such complex circumstances are simplified when "vibration effectiveness" at each frequency is plotted against the reciprocal of the TVR tension (in grams) because this relationship may imply effectiveness of vibratory energy along the parameter of frequency. In other words, such a relationship may be understood as a necessary vibratory input per motoneuronal firing because tension increases parallel to the number of EMG firings. This is shown in Fig. 4B, where the reciprocal of $T\left(\mathrm{~g}^{-1}\right)$ is plotted along the vertical axis and vibration frequency along the horizontal.

The results from three experiments are shown when eccentric weights of 8,12 and $16 \mathrm{~g}$ were used. The $1 / T-f$ relationship shows a downward shift as the 
eccentric weight become heavier as seen from the figure. It is very clear from the figure that heavier weights initiate TVR more effectively.

In our preceding reports in which cats under moderate chloralose-urethane anesthesia were used, it was demonstrated that relatively high amplitude vibration, around $10 \mathrm{~Hz}$, initiates motoneuronal discharges, the firing rate accurately corresponding to the mechanical vibration applied to the muscle tendon. When the vibration frequency is increased beyond this range, the frequency limiting behavior of the motoneuron was observed (Homma et al., 1970a). In other words, there was a "preferred frequency" of motoneuronal discharge rate above which the cell could not fire precisely even though more intense Ia bombardment rates were applied. The minimum vibratory amplitude which was necessary to elicit a "preferred frequency" was between $20-150 \mathrm{~Hz}$. The necessary minimum of the vibratory excursion along varied frequency was plotted as $\Delta L$ in the $\Delta L-f$ diagram in the preceding paper (Homma et al., 1970b). As already reported, a minimum value of $\Delta L$ was usally found near a $100 \mathrm{~Hz}$ vibration. This frequency was presented as the "optimal frequency" of muscle vibration. In the $\Delta L-f$ diagram, the area above $\Delta L-f$ curve is termed the "response area" of the motoneuron. This means that any vibratory stimuli that come within this area can cause motoneuronal excitation of a preferred frequency.

The same evaluation of $1 / T-f$ relationship was also done in the monkey experiment. All animals were under light anesthesia.

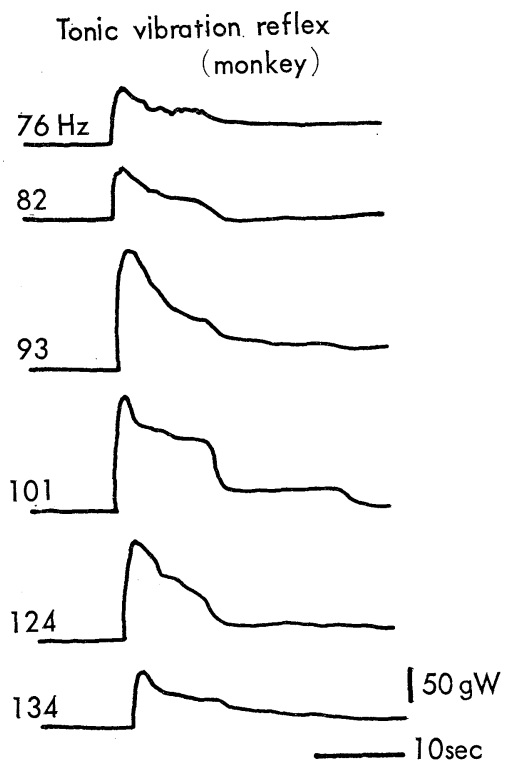

Fig. 5. TVR at various vibratory frequencies in monkey. TVR was smaller in both lower and higher ranges of vibratory frequency. 


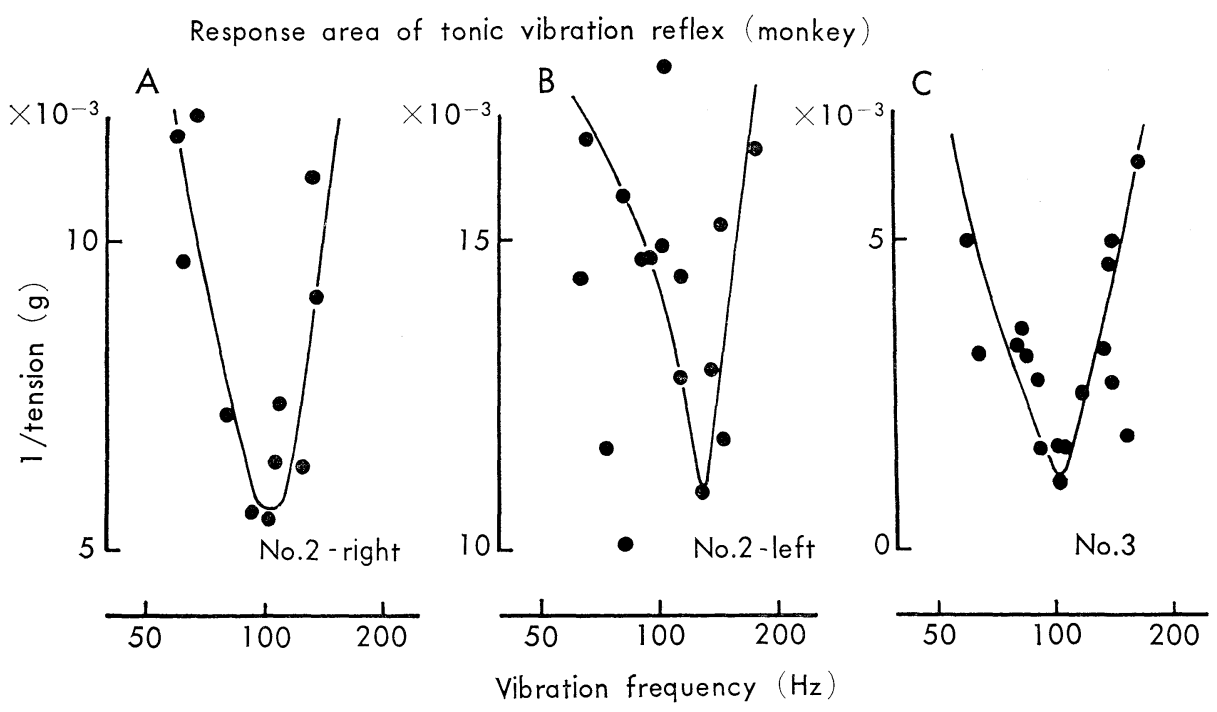

Fig. 6. Relation between reciprocals of maximum TVR tension and vibratory frequency in the monkey.

Figure 5 shows the actual recording of TVR in the monkey experiment. The maximum size of the TVR was attained relatively earlier (see Fig. 1B also) than in the human experiment, as already described. The reciprocals of maximum TVR are plotted against the vibratory frequency (Fig. 6).

The TVR of the monkey shown in this figure were measured just before it awakened from anesthesia. Since all the TVR tests required a span of minutes, this activity can be seen as more dispersion of the points in the diagram than in human experiment. However, the optimal frequency of each experiment in Figs. $6 \mathrm{~A}, \mathrm{~B}$ and $\mathrm{C}$ can be roughly estimated at 100,130 and $100 \mathrm{~Hz}$ respectively. Thus, this apparent discrepancy of the time course of TVR in human and monkey experiments had no influence upon the substantiation of the ubiquity of the "optimal frequency" characteristics of TVR.

Intervals of unitary EMG occur by the principle of integer multiplication of vibratory cyclic time, as seen in Figs. 2 and 3. Therefore it is assumed that TVR both in human subjects and monkeys are phenomena augmented by the increase in the EMG frequency according to the above principle. Since the maximum tension was observed at the optimal frequency, it means that the frequency of unitary EMG was highest at the optimal frequency. This will be discussed in detail later.

\section{DISCUSSION}

The same type of vibrator was used both in human and monkey experiments 
and tonic vibration reflex (TVR) could be initiated in both subjects, although the time course of each TVR was different in the human and monkey subjects. The TVR was always observed with an augmenting time course in human triceps surae muscle while in the monkey it rapidly reached its maximum size and gradually decayed to its "stationary state" of TVR. It was interesting that in the monkey experiment, TVR was most often observed when the animal was awakening, suggesting that some descending facilitating influences are essential for the initiation of TVR.

The gradual augmenting of TVR is shown to be effected by "vibratory facilitation" during which the interval of motoneuronal firing is shortened along vibratory frequencies. The relevant result upon which this part of discussion depends will be published elsewhere (Homma et al., 1971). However, the data available at present are still not enough to fully explain the phenomenon of motoneuronal firing interval shortening corresponding to the varied frequencies of vibratory stimulation. Forced stimulation of group Ia muscle afferent might well cause facilitation of the stretch reflex through posttetanic potentiation (LLOYD, 1949; Granit, 1956; Homma and KanO, 1962). Therefore, motoneuronal PTP which is caused by strong afferent bombardment during vibration can be considered as a probable support for the explanation of "vibratory facilitation."

The time course of TVR in monkeys was much different from that observed in human TVR. Monkey TVR reached its maximum value in the earliest period of the reflex and showed gradual decline, as seen in Fig. 1B. The considerable difference in their reflex mode is thought to be precipitated from the difference in the consciousness of the subjects, though the tonic activities of both groups were the same. It is well known that continued high frequency synaptic transmission is often followed by the Wedensky phenomenon or tetanic fade like that seen in the time course of the monkey TVR. Why then does the TVR time course of unanesthetized human subjects show a gradual recruitment or "vibratory facilitation"?

This may be considered in the following way. The $\mathrm{H}$-wave, a reliable sign for monosynaptic excitation of the human spinal cord, was shown to decrease during vibratory stimulation of the crural part, the skin afferent being considered necessary and a probable inhibitory inflow to the spinal reflex center (HAGBARTH and EKLUND, 1966a; LANCE et al., 1966; YAMANAKA, 1964). It is also known that continued excitation of the skin afferent elicits reflex activity of the flexors, which is a withdrawal reflex of its nature. It is possible that generalization of such withdrawal integration may play a role in the inhibition of the activity of extensor muscles. Such generalized tonic inhibition of the skin afferent may gradually decrease through a kind of adaptation phenomenon, and this may explain the gradual increase of human TVR fairly well. Thus, the specific time course of human TVR can be explained by gradual disinhibition. It is also surmised here that supraspinal influence may play a major part in such specific disinhibitory 
adaptation phenomenon, especially in the conscious human subject.

On the other hand, it is also known that stimulation of certain skin areas can have some facilitatory or inhibitory effects on gamma efferents (ELDRED and HAGBARTH, 1954). A gradual increase of TVR during vibratory stimulation may be due to an augmentation of gamma activity in the extensor muscle.

It was observed in the present investigation that the amount of TVR depended on the frequency of the vibration, the maximum vibratory effect being found near the "optimal frequency," as reported before. In our preceding report (HommA et al., 1970b), the $\Delta L$ of the $\Delta L-f$ relationship was determined for some constant number of motoneuronal firing when vibratory frequency and vibratory excursion $(\Delta L)$ were two parameters. The vibratory frequency at which minimum $\Delta L$ was attained was termed the "optimal frequency." As described before, the optimal frequency was found to exist at around $100 \mathrm{~Hz}$ vibration in the cat experiments. Such a range for the optimal frequency is also confirmed to exist near $100 \mathrm{~Hz}$ in the present experiment on human and monkey subjects.

\section{REFERENCES}

Bessou, P. and LAPORT, Y. (1962) Responses from primary and secondary endings of the same neuromuscular spindle of the tenuissimus muscle of the cat. In Symposium on Muscle Receptors, ed. by Barker, D. Hong Kong University Press, Hong Kong, 105-119.

Bianconi, R. and Van Der Meulen, J. P. (1963) The response to vibration of the end organs of mammalian muscle spindles. J. Neurophysiol., 26: 177-190.

Brown, M. C., Engberg, I. and Matthews, P. B. C. (1967) The relative sensitivity to vibration of muscle receptors of the cat. J. Physiol., 192: 773-900.

Eldred, E. and Hagbarth, K.-E. (1954) Facilitation and inhibition of gamma efferents by stimulation of certain skin areas. J. Neurophysiol., 17: 59-65.

Granit, R. (1956) Reflex rebound by post-tetanic potentiation. Temporal summationspasticity. J. Physiol., 131: 32-51.

Granit, R. and Henatch, H.-D. (1956) Gamma control of dynamic properties of muscle spindles. J. Neurophysiol., 19: 356-366.

Granit, R., Henatsch, H.-D. and Steg, G. (1956) Tonic and phasic ventral horn cells differentiated by posttetanic potentiation in cat extensors. Acta physiol. scand., 37: 114-126.

Hagbarth, K.-E. and Eklund, G. (1966a) Motor effects of vibratory muscle stimuli in man. In Muscular Afferents and Motor Control. Novel Symposium I, ed. by Granit, R. Almqvist and Wiksell, Stockholm, 177-186.

Hagbarth, K.-E. and Eklund, G. (1966b) Tonic vibration reffex (TVR) in spasticity. Brain Res., 2: 201-203.

Homma, S., Ishikawa, K. and Stuart, D. G. (1970a) Motoneuron responses to linearly rising muscle stretch. Am. J. Phys. Med., 49: 290-306.

Homma, S., Ishikawa, K. and Watanabe, S. (1967) Optimal frequency of muscle vibration for motoneuron firing. J. Chiba Med. Soc., 43: 190-196.

Homma, S., Kanda, K. and Watanabe, S. (1971) Monosynaptic coding of group Ia afferent discharges during vibratory stimulation of muscles. Jap. J. Physiol., 21: 405-417.

Homma, S. and KANO, M. (1962) Electrical properties of the tonic reflex arc in the human proprioceptive reflexes. In Symposium on Muscle Receptors, ed. by BARKER, D. Hong Kong University Press, Hong Kong, 167-174. 
Homma, S., Kobayashi, H. and Watanabe, S. (1970b) Vibratory stimulation of muscles and stretch reflex. Jap. J. Physiol., 20: 309-319.

Lance, J. W., DE Gail, P. and NeILson, R. D. (1966) Tonic and phasic spinal cord mechanisms in man. J. Neurol. Neurosurg. Psychiatry., 29: 535-544.

LLOYD, D. P. C. (1949) Post-tetanic potentiation of response in monosynaptic reflex pathways of the spinal cord. J. Gen. Physiol., 33: 147-170.

Matthews, P. B. C. (1966) The reflex excitation of the soleus muscle of the decerebrate cat caused by vibration to its tendon. J. Physiol., 184: 450-472.

YamanaKa, T. (1964) Effect of high frequency vibration on muscle spindle in the human body. J. Chiba Med. Soc., 40: 337-346. 